\title{
Asymmetric nuclear matter and neutron star properties
}

\author{
L. Engvik, M. Hjorth-Jensen and E. Osnes \\ Department of Physics, University of Oslo, N-0316 Oslo, Norway \\ G. Bao and E. Østgaard \\ Department of Physics, AVH, University of Trondheim, N-7055 Dragvoll, Norway
}

\begin{abstract}
We calculate properties of neutron stars such as mass and radius using a relativistic Dirac-Brueckner-Hartree-Fock approach. Modern meson-exchange potential models are used to evaluate the $G$-matrix for asymmetric nuclear matter. For pure neutron matter we find the maximum mass to be $M_{\max } \approx$ $2.4 M_{\odot}$ for a radius $R \approx 12 \mathrm{~km}$, whereas with a proton fraction of $30 \%$, we find $M_{\max } \approx 2.1 M_{\odot}$ for a radius $R \approx 10.5 \mathrm{~km}$, close to the experimental values. The implications are discussed.
\end{abstract}

PACS numbers: 97.60.Jd, 21.60.-n, 21.65.+f, 
The properties of neutron stars depend on the equation of state (EOS) at densities up to an order of magnitude higher than those observed in ordinary nuclei. Data on the EOS can be obtained from many sources, such as studies of the monopole resonance in finite nuclei, high-energy nuclear collisions, supernovae and neutron stars. Supernova simulations seem to require an EOS which is too soft to support some observed masses of neutron stars, whereas analyses of high-energy nuclear collisions indicate a rather stiff EOS, predicting neutron star masses which are too large. Thus, no definite statements can be made about the EOS at high densities, except that it should probably be moderately stiff in order to support maximum neutron star masses in a range from approximately $1.4 M_{\odot}$ to $1.9 M_{\odot}$ [1].

However, although quantitative calculations of the EOS for dense nuclear matter are still beset with many problems, there have recently been important changes in the qualitative picture. Several mechanisms have been studied, mechanisms which result in an EOS which is soft enough to support neutron stars with maximum masses in the range of the observed data. Among such processes we find exotic states of nuclear matter, such as kaon [2,3] or pion condensation [4]. Another scenario which gives neutron star masses within the experimental values, has been presented by Pethick and co-workers [5] [6]. These authors study properties of various phase transitions from spherical nuclei to uniform nuclear matter, and the coexistence of quark matter and nuclear matter over a finite fraction of the neutron star volume.

The scope of this work is to derive the EOS for asymmetric nuclear matter, using relativistic many-body theories within the framework of the Dirac-Brueckner-Hartree-Fock approach [7,8] and treating the Pauli operator which enters our formalism (see below) correctly. To our knowledge, this has not been done before. Asymmetric nuclear matter is important in e.g. studies of neutron star cooling, as demonstrated recently by Lattimer et al. [9] who showed that ordinary nuclear matter with a small asymmetry parameter can cool by the so-called direct URCA process even more rapidly than matter in an exotic state. In addition, at high densities, degrees of freedom represented by isobars and hyperons may also result in a rapid cooling of neutron stars [10]. 
Before we present our results, we briefly sketch below our calculational procedure, for further details see Ref. [11. Important ingredients in our calculations are the nucleonnucleon (NN) interaction which we take from meson-exchange models, and the renormalized NN potential in a medium like nuclear matter. The latter is accounted for by the reaction matrix $G$

$$
G(\omega)=V+V Q \frac{1}{\omega-Q H_{0} Q} Q G(\omega)
$$

where $\omega$ is the energy of the interacting nucleons, $V$ is the free NN potential, $H_{0}$ is the unperturbed energy of the intermediate scattering states, and $Q$ is the Pauli operator which prevents scattering into occupied states. The $G$-matrix is used in our many-body scheme described below in order to obtain the energy per particle in asymmetric nuclear matter. To calculate the NN potential, we use the Bonn A potential defined in table A.1 of Ref. [12]. This potential was shown by Brockmann and Machleidt [8], to reproduce the nuclear matter binding energy and saturation density within the framework of the Dirac-BruecknerHartree-Fock approach.

In order to test whether our EOS is appropriate for neutron stars, we evaluate the total mass and radius of such stars. Although there are recent relativistic calculations which address asymmetric nuclear matter, see e.g. the recent work of Huber et al. [13], one of the differences between this work and Ref. [13] is an exact treatment of the Pauli operator $Q$ for asymmetric nuclear matter. The conventional approach has been to extrapolate the results for symmetric nuclear matter and pure neutron matter [14]. Here we employ an exact expression for $Q$ in nuclear matter as described in e.g. Ref. [15 and calculate the energy per particle for asymmetric nuclear matter using an extended Brueckner-Hartree-Fock (BHF) method, see Ref. [16] for a discussion of the non-relativistic BHF theory, namely the DiracBrueckner-Hartree-Fock (DBHF) method. The DBHF method is a variational procedure, where the single-particle (sp) energies are obtained through an iterative self-consistency 
scheme. To describe the sp properties we depart from the Dirac equation for a free nucleon円, i.e.,

$$
(i \not \supset-m) \psi(x)=0
$$

where $m$ is the free nucleon mass and $\psi(x)$ is the nucleon field operator, which is conventionally expanded in terms of plane wave states and the Dirac spinors $u(p, s)$, and $v(p, s)$, where $p=\left(p^{0}, \mathbf{p}\right)$ is a four momentum and $s$ is the spin projection. The relativistic energy is $E(p)=\sqrt{m^{2}+|\mathbf{p}|^{2}}$. To account for medium modifications to the free Dirac equation, we introduce the notion of the self-energy $\Sigma(p)$, which for nucleons can be written as

$$
\Sigma(p)=\Sigma_{S}(p)-\gamma_{0} \Sigma^{0}(p)+\gamma \mathbf{p} \Sigma^{V}(p)
$$

The momentum dependence of $\Sigma^{0}$ and $\Sigma_{S}$ is rather weak [8.17]. Moreover, $\Sigma^{V}<<1$, and it is customary to rewrite the self-energy as

$$
\Sigma \approx \Sigma_{S}-\gamma_{0} \Sigma^{0}=U_{S}+U_{V}
$$

where $U_{S}$ is an attractive scalar field and $U_{V}$ is the time-like component of a repulsive vector field. The finite self-energy modifies the free Dirac spinors as

$$
\tilde{u}(p, s)=\sqrt{\frac{\tilde{E}(p)+\tilde{m}}{2 \tilde{m}}}\left(\begin{array}{c}
\chi_{s} \\
\frac{\boldsymbol{\sigma} \cdot \mathbf{p}}{\tilde{E}(p)+\tilde{m}} \chi_{s}
\end{array}\right),
$$

where we let the terms with tilde represent the medium modified quantities. Here we have defined [8, [17]

$$
\tilde{m}=m+U_{S},
$$

and

\footnotetext{
${ }^{1}$ Hereafter we set $G=c=\hbar=1$, where $G$ is the gravitational constant.
} 


$$
\tilde{E}_{\alpha}=\tilde{E}\left(p_{\alpha}\right)=\sqrt{\tilde{m}^{2}+\mathbf{p}_{\alpha}^{2}}
$$

and the sp potential is given by

$$
u_{\alpha}=\sum_{h \leq k_{F}} \frac{\tilde{m}^{2}}{\tilde{E}_{h} \tilde{E}_{\alpha}}\left\langle\alpha h\left|G\left(\omega=\tilde{\varepsilon}_{\alpha}+\tilde{\varepsilon}_{h}\right)\right| \alpha h\right\rangle_{\mathrm{AS}},
$$

where $G$ is the relativistic $G$-matrix [8]. We can also express the sp potential in terms of the constants $U_{S}$ and $U_{V}$,

$$
u_{\alpha}=\frac{\tilde{m}}{\tilde{E}_{\alpha}} U_{S}+U_{V}
$$

The sp energies $\varepsilon$ can then be written as

$$
\tilde{\varepsilon}_{\alpha}=\tilde{E}_{\alpha}+U_{V}
$$

where we have used the continuous choice for the single-particle energies [16].

Eqs. (8)-(10) are solved self-consistently starting with adequate values for the scalar and vector components $U_{S}$ and $U_{V}$. This iterative scheme is continued until these parameters show little variation. The calculations are carried out in the nuclear matter rest frame, avoiding thereby a cumbersome transformation between the two-nucleon center-of-mass system and the nuclear matter rest frame. The additional factors $\tilde{m} / \tilde{E}$ in the above equations arise due to the normalization of the neutron matter spinors $\tilde{w}$, i.e. $\tilde{w}^{\dagger} \tilde{w}=1[8]$. With the obtained sp energies, we can calculate the relativistic energy per particle, see e.g. Refs. 8.11]. All relevant equations with a given proton-neutron fraction are given in Ref. [15].

With these preliminaries, we present our results for the energy per particle with various proton fractions in Fig. 1 1. For relatively small proton fractions, the energy per particle exhibits much the same curvature as the curve for pure neutron matter at high densities, although the energy per particle is less repulsive at high densities. At lower densities, the situation is rather different. This is due to the contributions from various isospin $T=0$ partial waves, especially the contribution from the ${ }^{3} S_{1^{-}}{ }^{3} D_{1}$ channel, where the tensor force component of the nucleon-nucleon potential provides additional binding. From Fig. 11 we note that with a proton fraction of $15 \%$, the energy per particle starts to become attractive 
at low densities (in the region $0.07 \mathrm{fm}^{-3}$ to $0.3 \mathrm{fm}^{-3}$ ). For larger proton fractions, additional attraction to the energy per particle is introduced. The next step in our calculations is thus to evaluate the EOS and the total mass and radius of a neutron star from the above energies per particle with the proton fractions shown in Fig. 1, in order to see how different proton fractions influence the mass and radius of neutron stars. Here we assume that the neutron stars we study exhibit an isotropic mass distribution. Hence, from the general theory of relativity, the structure of a neutron star is determined through the Tolman-OppenheimerVolkov equation, i.e.

$$
\frac{d P}{d r}=-\frac{\{\rho(r)+P(r)\}\left\{M(r)+4 \pi r^{3} P(r)\right\}}{r^{2}-2 r M(r)},
$$

where $P(r)$ is the pressure and $M(r)$ is the gravitional mass inside a radius $r$. To obtain observables like the mass and radius of a neutron star, we combine Eq. (11) with the equation of state (EOS), which is defined as $P(n)=n^{2}(\partial \epsilon / \partial n)$, where $\epsilon=\mathcal{E} / A$ is the energy per particle and $n$ is the particle density. Our EOS is valid in a limited density range from 0.1 $\mathrm{fm}^{-3}$ to $0.8 \mathrm{fm}^{-3}$. It is therefore coupled to other equations of state at higher and lower densities as outlined in Ref. [11]. Total masses and radii are calculated and parametrized as functions of the central density $n_{c}$. These results are presented in Figs. 2 and 3 , for the total mass and radius, respectively.

The neutron star equation of state should probably be only moderately stiff to support maximum neutron star masses of only $1.9 M_{\odot}$ [1]. From Figs. 2 and 3 we see that our relativistic EOS for pure neutron matter seems to be too stiff, since it gives a predicted maximum mass of $M_{\max } \approx 2.4 M_{\odot}$ with a corresponding radius of $R=12 \mathrm{~km}$. However, the EOS for neutron star matter could be softened considerably due to pion or kaon condensation because of the lower symmetry energy of nuclear matter, and maximum masses are then reduced correspondingly from the cases with no condensates. We see from Figs. 2 and 3 that for our EOS the calculated maximum mass can be reduced to $M_{\max } \approx 2.0 M_{\odot}$ with a corresponding radius of $R \approx 10 \mathrm{~km}$. Pions may be likely to condense in neutron star matter because neutrons at the top of the Fermi sea could decay to protons plus electrons [4], and 
kaon condensation is also believed to be a possible mechanism which could be energetically favorable in the interior of neutron stars [3, 18, 19]. Both pion and kaon condensation would then increase the proton abundance in the matter, possibly up to more than $40 \%$ protons, i.e. close to symmetric nuclear matter, and produce a softer EOS and smaller maximum mass. Our results show this, if we assume that e.g. kaon condensation is a likely mechanism, an increased proton fraction results in smaller masses. Our EOS, even with a proton fraction close to symmetric nuclear matter, results in maximum masses which are slightly above the experimental values [1]. However, our calculation of the EOS is to first order in the reaction matrix $G$, and we would therefore expect that higher-order many-body contributions to soften the EOS further. This was indeed shown in a preliminary study for symmetric nuclear matter by Jiang et al. [20]. Although only a set of higher-order contributions was considered, the above authors obtain a softening of the relativistic EOS. The study of such effects will be studied by us in future works. 


\section{REFERENCES}

[1] S. E. Thorsett, Z. Arzoumanian, M. M. McKinnon and J. H. Taylor, Astrophys. J. 405, L29 (1993)

[2] D. B. Kaplan and A. E. Nelson, Phys. Lett. B175, (1987) 57

[3] G. E. Brown, in proceedings of Perspectives in Nuclear Structure, Niels Bohr Institute, June 14-19, 1993, Nucl. Phys. A in press

[4] R. Mittet and E. Østgaard, Phys. Rev. C 37, 1711 (1988)

[5] C. P. Lorenz, D. G. Ravenhall and C. J. Pethick, Phys. Rev. Lett. 70, 379 (1993)

[6] H. Heiselberg, C. J. Pethick and E. F. Staubo, Phys. Rev. Lett. 70, 1355 (1993)

[7] L.S. Celenza and C.M. Shakin, Relativistic Nuclear Physics: Theories of Structure and Scattering, Vol. 2 of Lecture Notes in Physics, (World Scientific, Singapore, 1986)

[8] R. Brockmann and R. Machleidt, Phys. Rev. C 42, 1965 (1990)

[9] J. M. Lattimer, C. J. Pethick, M. Prakash and P. Haensel, Phys. Rev. Lett. 66, (1991) 2701

[10] M. Prakash, M. Prakash, J. M. Lattimer and C. J. Pethick, Astrophys. J. 390, L77 (1992)

[11] G. Bao, L. Engvik, M. Hjorth-Jensen, E. Osnes and E. Østgaard, Nucl. Phys. A in press

[12] R. Machleidt, Adv. Nucl. Phys. 19, 189 (1989)

[13] H. Huber, F. Weber and M. K. Weigel, Phys. Lett. B317, 485 (1993); preprint (1994)

[14] I. Bombaci and U. Lombardo, Phys. Rev. C 44, 1892 (1991)

[15] H. Q. Song, Z. X. Wang and T. T. S. Kuo, Phys. Rev. C 46, 1788 (1992)

[16] C. Mahaux, P. F. Bortignon, R. A. Broglia and C. H. Dasso, Phys. Rep. 120, 1 (1985) 
[17] B. D. Serot and J. D. Walecka, Adv. Nucl. Phys. 16, 1 (1986)

[18] V. Thorsson, M. Prakash and J. M. Lattimer, Nucl. Phys. A572, 693 (1994)

[19] G. E. Brown, C.-H. Lee, M. Rho and V. Thorsson, NORDITA preprint no. 30 (1993)

[20] M. F. Jiang, R. Machleidt and T. T. S. Kuo, Phys. Rev. C 47, 2661 (1993) 


\section{FIGURES}

FIG. 1. The energy per particle for asymmetric nuclear matter as function of the particle density for different proton fractions. 
FIG. 2. $M / M_{\odot}$ for various proton fractions as function of the central density $n_{c}$. 
FIG. 3. The total radius $R$ for different proton fractions as function of the central density $n_{c}$. 
This figure "fig1-1.png" is available in "png" format from: http://arxiv.org/ps/nucl-th/9406028v1 
This figure "fig1-2.png" is available in "png" format from: http://arxiv.org/ps/nucl-th/9406028v1 
This figure "fig1-3.png" is available in "png" format from: http://arxiv.org/ps/nucl-th/9406028v1 